\title{
Removal of Phosphorus in Metallurgical Silicon by Rare Earth Elements
}

\author{
KAI TANG, OLE MARTIN LØVVIK, JAFAR SAFARIAN, XIANG MA, \\ and MERETE TANGSTAD
}

\begin{abstract}
Removal of phosphorus in metallurgical silicon is one of the crucial steps for the production of solar grade Si feedstock. The possibility of doping rare earth elements for phosphorus removal has in this work been studied both theoretically and experimentally. Thermochemical properties of $\mathrm{Ce}, \mathrm{Nd}$, and $\mathrm{Pr}$ monophosphides have first been estimated by ab initio thermodynamic simulations based on density functional theory and the direct phonon method. The reliability of the first principles calculations was assessed by coupling with the phase diagram data of the Pr-P system. Equilibrium calculations confirmed the existence of stable rare earth monophosphides in solid silicon. Experimental investigations were then carried out, employing a high temperature resistance furnace. The Ce-doped silicon samples were examined by electron probe micro analyzer and inductively coupled plasma analysis. The efficiency of phosphorus removal by means of rare earth doping was discussed in detail in the paper.
\end{abstract}

DOI: $10.1007 / \mathrm{s} 40553-014-0025-6$

(C) ASM International (ASM) and The Minerals, Metals \& Materials Society (TMS) 2014

\section{INTRODUCTION}

REMOVAL of phosphorus in metallurgical silicon is one of the crucial steps for the production of solar grade $\mathrm{Si}(\mathrm{SoG}-\mathrm{Si}$ ) feedstock. Great effort has been made in order to develop the processes for effective phosphorus removal in silicon. It has recently been shown by Meteleva-Fischer ${ }^{[1]}$ that the rare earth elements are able to form stable phosphides in pure silicon. The rare earth elements also possess high removal efficiency for boron and phosphorus comparable to that of calcium, and remove aluminum better than calcium. The segregation coefficients of rare earth elements in silicon are in the order of magnitude of $10^{-4}$, which indicates better selfremoval from silicon matrix than that of calcium.

The possibility of doping rare earth elements for phosphorus removal has been examined both theoretically and experimentally. Thermochemical properties of the cerium and praseodymium monophosphides were first evaluated using $a b$ initio calculations. The phase equilibria in the Pr-P and Ce-P systems were then accessed by the Calphad approach. A thermochemical description of the Si-rich part of the Si-C-Ce-P system has thus been constructed. The phase transformations during the solidification of the Ce-doped Si-P alloys can be predicted using the thermodynamic description of the $\mathrm{Si}-\mathrm{C}-\mathrm{Ce}-\mathrm{P}$ system. Based on the calculation results, two

KAI TANG, Senior Research Scientist, and JAFAR SAFARIAN, Research Scientist, are with the SINTEF Materials and Chemistry, Trondheim, Norway. Contact e-mail: kai.tang@sintef.no OLE MARTIN LØVVIK, Senior Research Scientist, and XIANG MA, Research Scientist, are with the SINTEF Materials and Chemistry, Oslo, Norway. MERETE TANGSTAD, Professor, is with the Department of Materials Science and Technology, Norwegian University of Science and Technology, Trondheim, Norway.

Manuscript submitted October 30, 2013.

Article published online August 5, 2014 sets of experiments were carried out. The precipitation of cerium phosphides was observed in the silicon crystal boundaries from the electron probe microanalyzer (EPMA) examination and inductively coupled plasma mass spectrometry (ICP-MS) analysis.

\section{THEORETICAL CONSIDERATION}

The formation of stable rare earth phosphide in metallurgical silicon with very dilute concentration of phosphorus is dependent essentially upon the chemical potential in the Si-RE-P (RE: rare earth element) system. It has already been confirmed that the cerium monophosphide, $\left.{ }^{[2,} 3\right]$ having the fcc- $\mathrm{NaCl}$ structure, exists in the Ce-P system. However, neither phase diagram nor thermodynamic date exists for this binary system. For the rare earth-phosphorus phase diagrams, only the Pr-P binary was reported by Mironov. ${ }^{[4]}$ In addition to the phase equilibrium information, thermodynamic properties of EuP and GdP were also reported in the literature. ${ }^{[5]}$ The Gibbs energies of EuP and GdP show large negative values in the temperature range of interest. The monophosphides of rare earth also show congruent melting behavior at rather high temperatures, in general higher than $2773 \mathrm{~K}\left(2500^{\circ} \mathrm{C}\right)$. The high melting temperatures of rare earth monophosphides imply the possibility of precipitation of monophosphides in silicon with relatively low concentration of phosphorus.

The Gibbs energy of praseodymium monophosphide, PrP, can be assessed from the measured phase diagram data. Figure 1 shows the calculated phase equilibria in the Pr-P system. Experimental points reported by Mironov $^{[4]}$ are also superimposed.

The first principles calculations were performed using the Vienna ab initio simulation package (VASP) $)^{[6,7]}$ 


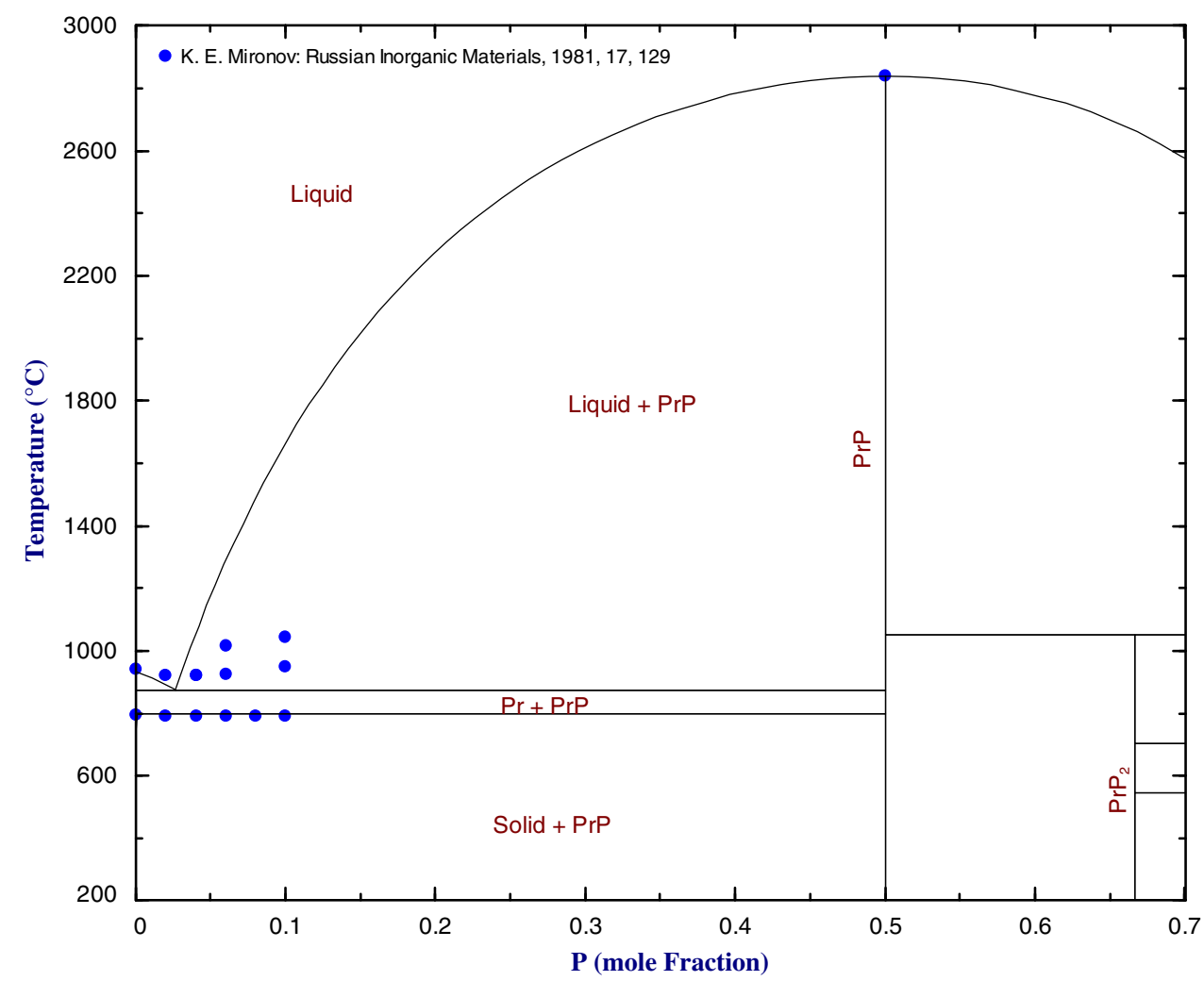

Fig. 1-The new assessed Pr-P phase diagram.

within the $\mathrm{PBE}^{[8]}$ implementation of the generalized gradient approximation. Standard functional based on the projector augmented wave method was employed; the valence electron configuration was thus $4 f^{1} .5 d^{9} .6 s^{2}$ for $\mathrm{Ce}, 4 f^{4} .5 d^{8} .6 \mathrm{~s}^{2}$ for $\mathrm{Nd}$, and $3 s^{2} .3 p^{3}$ for $\mathrm{P}$. The cut-off energy for the plane wave basis function expansion was $500 \mathrm{eV}$, and the distance between $k$ points in the numerical grids in reciprocal space was always less than $0.25 \AA^{-1}$. The criterion for self-consistency in the Kohn-Sham iterations was that total energies and band energies differed by less than $10^{-5} \mathrm{eV}$ between two consecutive iterations. The electron occupancy was smeared with a Gaussian scheme with a breadth of $0.2 \mathrm{eV}$; this was extrapolated to zero when calculating the total energies. The overall numerical convergence of relative energies was in the order of $1 \mathrm{meV}$ from the abovementioned numerical parameters.

Both CeP and NdP display a rock-salt crystal structure within the $\mathrm{Fm}_{-} 3 m$ space group. The experimental crystal structures were used as input for structural relaxation in VASP. The residual minimization scheme with direct inversion in the iterative subspace was used, which is a quasi-Newton algorithm. The condition for a relaxed structure was that the remaining forces were less than $0.005 \mathrm{eV} / \AA$. Both interatomic forces and internal stress were relaxed simultaneously.

Phonon calculations were calculated using the Phonon software, ${ }^{[9]}$ where the direct method may be used to calculate thermodynamic variables as function of temperature. This relies on the harmonic approximation, leading to the lattice partition function when all non- equivalent phonon modes have been calculated. A super cell of $2 \times 2 \times 2$ conventional cubic unit cells was used for both $\mathrm{CeP}$ and $\mathrm{NdP}$, each consisting of 32 atoms. Similarly super cells of $\mathrm{Ce}, \mathrm{Nd}$, and $\mathrm{P}$ were used for phonon calculations, consisting of 48,72 , and 48 atoms, respectively. This ensured that all lattice constants were larger than $11 \AA$, so that interaction between periodic images of displaced atoms was negligible.

The thermodynamic functions of $\mathrm{CeP}$ and $\mathrm{NdP}$ as calculated by VASP and the Phonon software were quite similar, and only the functions of $\mathrm{CeP}$ are shown in Figure 2. We note that Gibbs energy of formation (using the standard states of the elements as reference point) turns negative around $250 \mathrm{~K}\left(-23{ }^{\circ} \mathrm{C}\right)$ for both $\mathrm{NdP}$ and CeP. It is increasingly negative for higher temperatures, which points to the stability of $\mathrm{CeP}$ and $\mathrm{NdP}$ at elevated temperature.

Figure 3 compares the calculated Gibbs energies of the rare earth monophosphides and calcium phosphide. Generally speaking, the calculated G-T relations are rather similar for all four rare earth monophosphides. The rare earth monophosphides are more stable than the calcium phosphide, $\mathrm{Ca}_{3} \mathrm{P}_{2}$, particularly at high temperatures. The $G_{\mathrm{CeP}}$ values obtained from the $a b$ initio calculation are approximately $300 \mathrm{~kJ} / \mathrm{mol}$ higher than those of $G_{\mathrm{EuP}}$ and $G_{\mathrm{GdP}}$. The $G_{\mathrm{PrP}}$ values evaluated from the phase diagram data are also about $100 \mathrm{~kJ} / \mathrm{mol}$ higher than those of $G_{\mathrm{EuP}}$ and $G_{\mathrm{GdP}}$. Since the physiochemical properties of lanthanide series elements are rather similar, the Gibbs energy of CeP needs to be re-evaluated assuming the similar melting behavior 


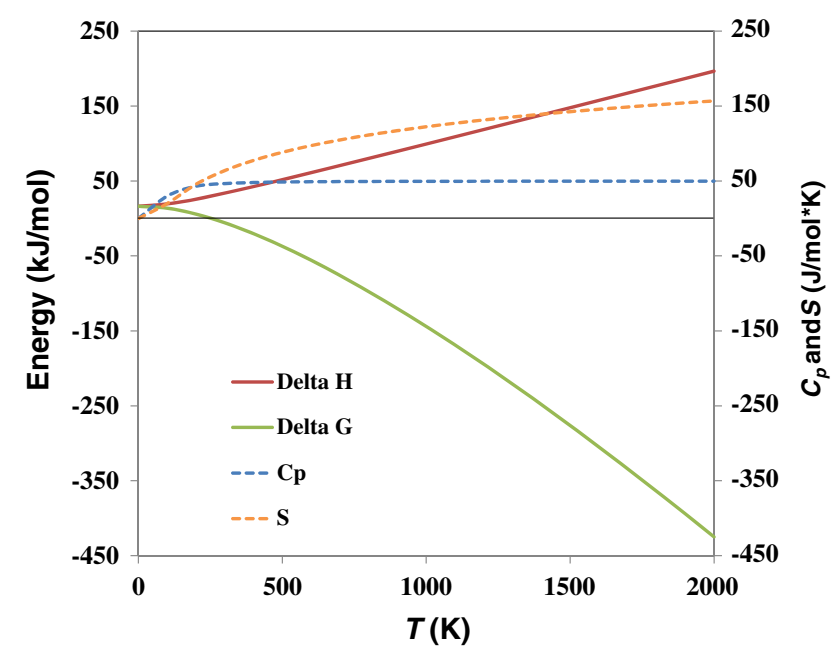

Fig. 2-The thermodynamic functions of $\mathrm{CeP}$ as a function of temperature calculated by VASP and the Phonon software.

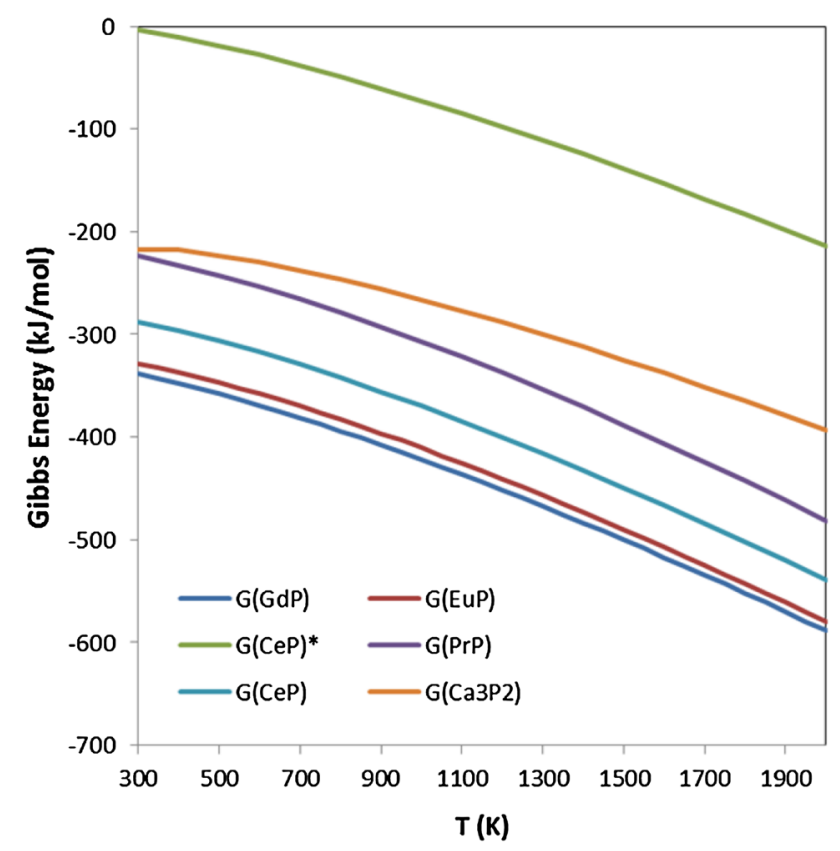

Fig. 3-The Gibbs energies of rare earth monophosphides and $\mathrm{Ca}_{3} \mathrm{P}_{2}$.

of cerium phosphide in the Ce-P binary. The relatively large difference between the $a b$ initio results and the phase diagram assessment is likely due to the choice of the standard states of the elements as reference point in the former calculations. The temperature dependence is very similar in the two cases, which indicates that the reference energy is the main difference.

Figure 4 is the tentative Ce-P phase diagram proposed by the present authors. The reassessed Gibbs energy of $\mathrm{CeP}$ is shown in Figure 3, which is about $300 \mathrm{~kJ} / \mathrm{mol}$ lower than the values calculated by ab initio method.

In order to evaluate the P-removal by formation of the cerium monophosphide, a thermodynamic description of the $\mathrm{Ce}-\mathrm{Si}$ system is also essential. Phase relations in the Ce-Si system were determined experimentally by Bulanova et al. ${ }^{[10]}$ Thermodynamic descriptions of the $\mathrm{Ce}-\mathrm{Si}$ system were reported by Grobner et al. ${ }^{[11]}$ and Shukla et al. ${ }^{[12]}$ The former one was used in the present study for the Redlich-Kister parameters of the liquid phase, although the latter one using the modified quasichemical model fits experimental data better. The existence of $\mathrm{CeSiP}_{3}$ was reported by Hayakawa et $a l^{[13]}$ However, it has not been considered in the present study due mainly to the lack of experimental thermochemical properties. Because the graphite crucible was used in the present experiments, thermodynamic description of the $\mathrm{Ce}-\mathrm{C}$ system was considered in the present study and taken from Peng et al. ${ }^{[14]}$

Using the above-assessed subsystems, a thermodynamic description for the Si-based Si-C-Ce-P system has been constructed and used to predict the phase transformation during solidification of Ce-doped Si-P alloys. Figure 5 shows the calculated phase transition of a Si30 ppmwP-1wt pctCe alloy. Obviously, the precipitation of cerium monophosphide, $\mathrm{CeP}$, is possible in the temperature range $703 \mathrm{~K}-1203 \mathrm{~K} \quad\left(430{ }^{\circ} \mathrm{C}-930{ }^{\circ} \mathrm{C}\right)$, under the so-called Scheil-solidification condition at cooling rate $10 \mathrm{~K} / \mathrm{min}$. This means that the formation of $\mathrm{CeP}$ requires relative high contents of $\mathrm{Ce}$ and $\mathrm{P}$ in liquid silicon phase. Therefore, the precipitation of $\mathrm{CeP}$ occurs closed to the end of solidification.

\section{EXPERIMENTAL}

Experimental verifications were carried out in a graphite resistance furnace in the SINTEF/NTNU laboratory. Detailed descriptions of the experimental apparatus can be found elsewhere. ${ }^{[15]}$ Alloys were first prepared by mixing of the Si-0.1wt petP and Si-0.05wt petB master alloys with electronic grade silicon (EG-Si). Analytical grade cerium powder ( $99.9 \mathrm{wt}$ pct) was added as dopant to the premelted alloys. For each experiment, about 1 mass present of Ce was doped. The samples were placed in high purity graphite crucibles and heated to $1773 \mathrm{~K}$ $\left(1500^{\circ} \mathrm{C}\right)$ for about 60 minute under the pure $\operatorname{Ar}(5 \mathrm{~N})$ atmosphere. The crucible was then cooled down to the room temperature by either the fixed cooling rate $(10 \mathrm{~K} /$ min) or natural cooling. The graphite crucibles were cracked in the experiments with controlled cooling rate at $10 \mathrm{~K} / \mathrm{min}$, whereas the samples of natural cooling were successfully obtained. So, only the samples of natural cooling are used in this work.

The crucibles were filled with epoxy resin and cross sectioned vertically. The cross sections were trimmed and ground to fit the mould for the microprobe analysis. The boron, cerium, phosphorus, and calcium contents of materials before and after experiments were determined by ICP-MS.

\section{RESULTS}

The contents of cerium, phosphorus, and boron in the two silicon metal samples after solidification to room temperature as determined by ICP-MS analysis, are 


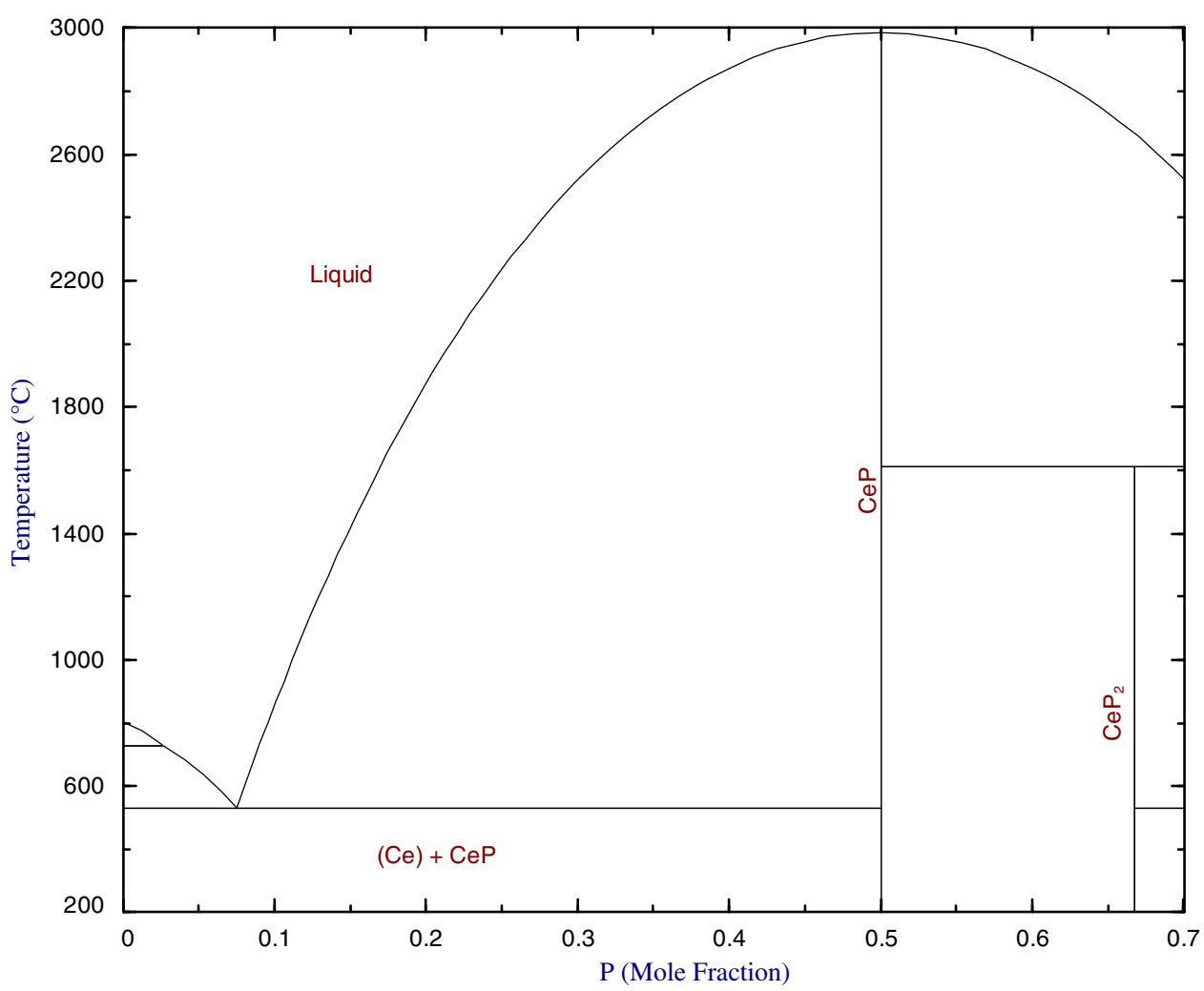

Fig. 4-The tentative phase diagram of Ce-P system.

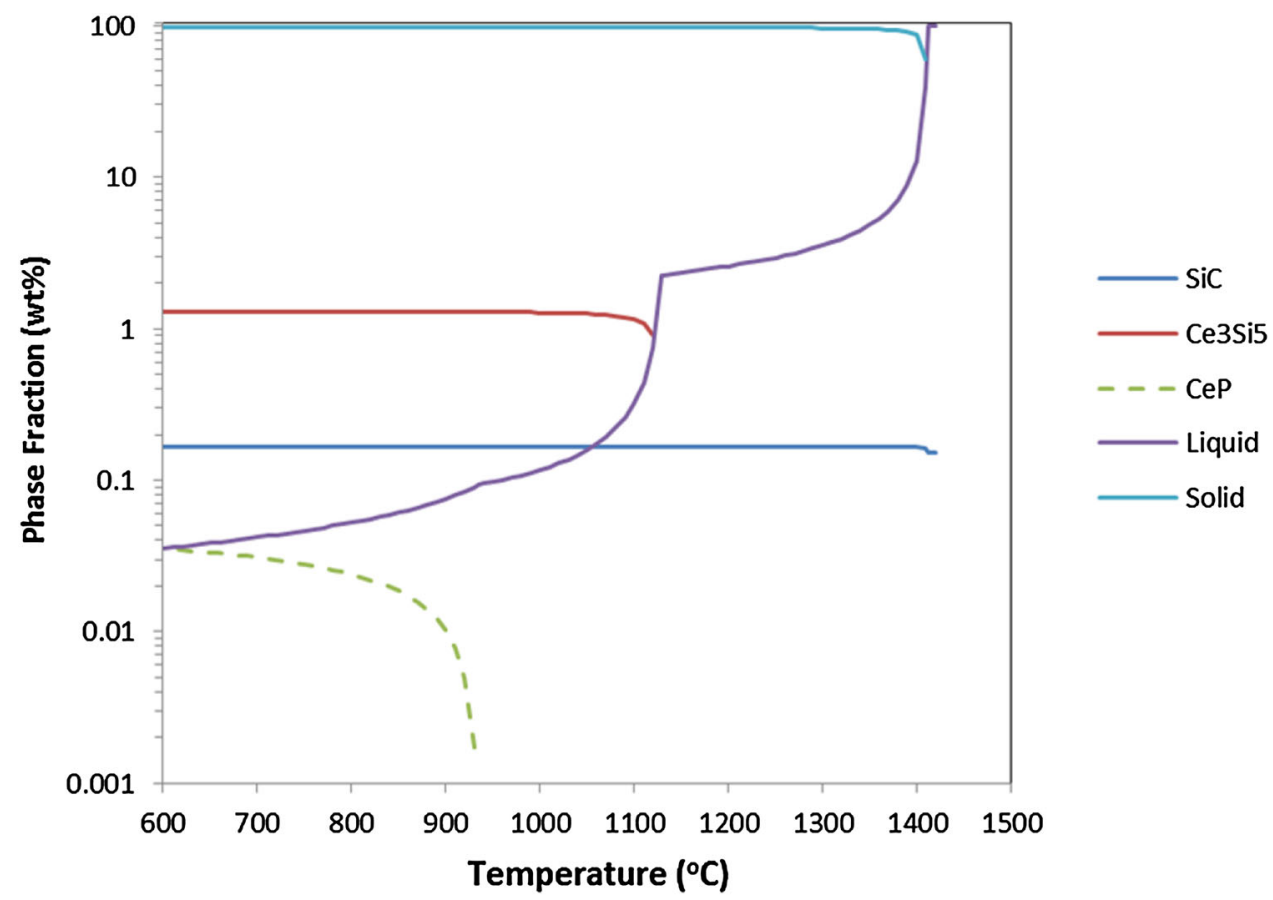

Fig. 5-The predicted phase precipitation during solidification of Ce-doped Si-30(ppmw)P metal. 
listed in following Table I. The contents of $\mathrm{Ca}$ in the sample are probably from cerium, since the pure cerium is generally produced by metallothermic reduction.

Although the content of cerium in silicon was aimed at $1 \mathrm{wt}$ pct, the actual Ce contents in two samples are much lower than the target value. It is probably that the added pure cerium dopant is oxidized during heating of the samples.

Figure 6 shows the EPMA picture of the sample 1. Figure 7 shows the area of microprobe analysis in Sample 1. Clearly, the formation of cerium phosphide, rather than the $\mathrm{CeSiP}_{3}$, can be confirmed from the EPMA analysis. Even though the Ca contents in sample are approximately the same as $\mathrm{Ce}$, the $\mathrm{Ca}_{3} \mathrm{P}_{2}$ formation in grain boundary is not observed from the picture. Since the amounts of $\mathrm{P}$ and $\mathrm{Ce}$ are rather lower, the results of EPMA analysis may need to be further verified by advanced analytical method, for instance, the secondary ion mass spectrometry.

Table I. Contents of Impurities in SoG-Si Samples

\begin{tabular}{lcccc} 
Ce $(\mathrm{ppm})$ & Other REEs $(\mathrm{ppm})$ & $\mathrm{B}(\mathrm{ppm})$ & $\mathrm{P}(\mathrm{ppm})$ & $\mathrm{Ca}(\mathrm{ppm})$ \\
\hline 194 & 0.22 & 3.3 & 14 & 164 \\
145.4 & 0.28 & 0.1 & 22.5 & 153 \\
\hline
\end{tabular}

The segregation coefficient of cerium in pure silicon can also be estimated using the current evaluated thermodynamic description. At $1685 \mathrm{~K}\left(1412{ }^{\circ} \mathrm{C}\right)$, the

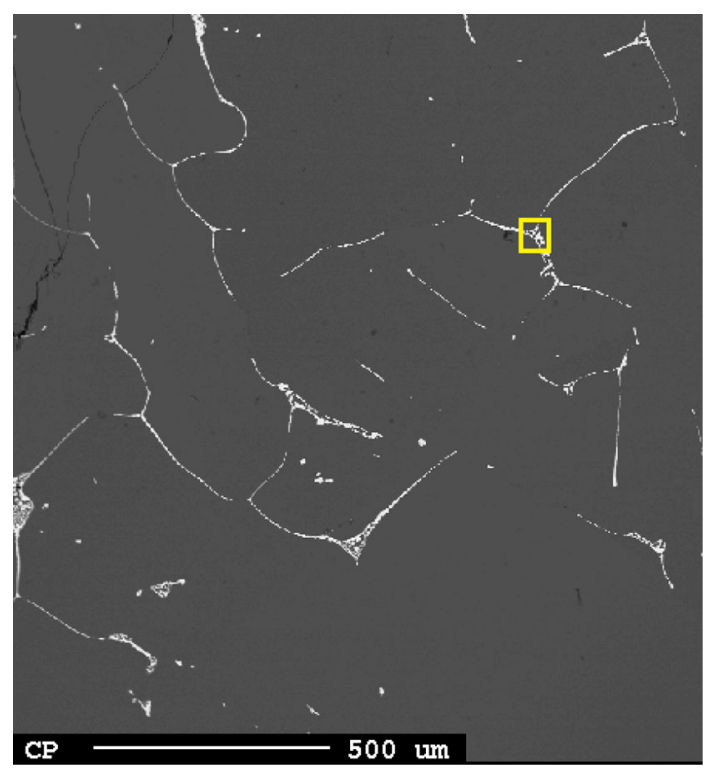

Fig. 7-SME picture of Ce-doped Si sample.



Fig. 6-EMPA picture of Ce-doped Si sample. 
segregation coefficient of cerium is $1.2 \times 10^{-4}$, which is in good agreement with the value for La, being $1-2 \times 10^{-4}$, estimated by Meteleva-Fischer. ${ }^{[1]}$

\section{CONCLUSIONS}

The possibility of doping rare earth elements for phosphorus removal has in this paper been studied both theoretically and experimentally. Thermochemical properties of cerium monophosphide have first been calculated using $a b$ initio thermodynamic simulations based on density functional theory and the direct phonon method. Coupling with the experimental Pr-P phase diagram data, thermodynamic properties of cerium monophosphide have been assessed. Phase equilibria in the Ce-P system were then predicted using the Calphad approach. A thermodynamic description of the Si-rich Si-C-Ce-P system has thus been constructed.

The phase transformations during the solidification of the Ce-doped Si-P alloys can be predicted using the thermodynamic description of the Si-C-Ce-P system. Based on the calculation results, experimental verifications were carried out. The precipitation of cerium phosphides was observed in the silicon crystal boundaries from the EPMA examination and ICP-MS analysis. It is confirmed both theoretically and experimentally that cerium possess the high removal efficiency for phosphor comparable to that of calcium. The segregation coefficient of cerium in silicon is around $1.2 \times 10^{-4}$, which indicates the good ability of selfremoval from silicon matrix.

\section{ACKNOWLEDGMENTS}

The present work was funded by the Research Council of Norway (contract no. 208363) in the ReSi$\mathrm{Na}$ project.

\section{REFERENCES}

1. Y. Meteleva-Fischer: Alloying Refining of Metallurgical Grade Silicon with Rare Earth Elements, TMS 2013, The Minerals, Metals \& Materials Society San Antonia, TX, 2013.

2. I. Vedel, A.M. Redon, J. Rossat-Mignod, O. Vogt, and J.M. Leger: J. Phys. C: Solid State Phys., 1987, vol. 20 (23), p. 3439.

3. H. Bartholin, D. Florence, G. Parisot, J. Paureau, and O. Vogt: Phys. Lett. A, 1977, vol. 60 (1), pp. 47-49.

4. K.E. Mironov: Russian Inorg. Mater., 1981, vol. 17 (2), pp. 129 32.

5. L.B. Pankratz: Thermodynamic Properties of Carbides, Nitrides, and other Selected Substances, U.S. Department of the Interior, Washington, DC, 1995.

6. G. Kresse and J. Furthmüller: Phys. Rev. B, 1996, vol. 54 (16), pp. 11169-86.

7. G. Kresse and J. Hafner: Phys. Rev. B, 1993, vol. 47 (1), pp. 558-61.

8. J.P. Perdew, K. Burke, and M. Ernzerhof: Phys. Rev. Lett., 1996, vol. 77 (18), pp. 3865-68.

9. K. Parlinski, Z.Q. Li, and Y. Kawazoe: Phys. Rev. Lett., 1997, vol. 78 (21), pp. 4063-66.

10. M.V. Bulanova, P.N. Zheltov, K.A. Meleshevich, P.A. Saltykov, and G. Effenberg: J. Alloy. Compd., 2002. vol. 345 (1-2), pp. 110-15.

11. J. Grobner, D. Mirkovic, and R. Schmid-Fetzer: Metall. Mater. Trans. A, 2004, vol. 35A, pp. 3349-62.

12. A. Shukla, Y.B. Kang, and A.D. Pelton: Int. J. Mater. Res., 2009, vol. 100 (2), pp. 208-17.

13. H. Hayakawa, K. Nomura, and S. Ono: Nippon Kagaku Kaishi, 1978, vol. 9, p. 1200.

14. Y. Peng, Y. Du, L. Zhang, C. Sha, S. Liu, F. Zheng, D. Zhao, X. Yuan, and L Chen: CALPHAD, 2011, vol. 35 (4), pp. 533-41.

15. L.K. Jakobsson and M. Tangstad: International Smelting Technology Symposium, Wiley, 2012, pp. 179-84. 